\title{
Multithreshold Voltage Technology for Low Power Bus Architecture
}

\author{
A. Rjoub, O. Koufopavlou \\ VLSI Design Laboratory \\ Department of Electrical \& Computer Engineering \\ University of Patras \\ 6500 Patras - GREECE
}

Key words: Multithreshold Voltage, Low Swing, Low Power Design, Bus Architecture.

\begin{abstract}
Multithreshold voltage technology for low swing voltage bus architecture is proposed. Three new different classes of driver/repeater/receiver circuits are introduced. In the driver circuits, high threshold MOSFET transistor have been inserted in order to decrease its output swing voltage. To re-pull up the low swing to full swing voltage, innovated low delay cross-coupled latch circuit receivers are proposed. The same architecture with new repeater circuits based on multithreshold voltage are introduced in order to drive internal long interconnection lines and decreasing the total delay time. For $2 \mathrm{~V}$ supply voltage using 0.5 m process technology, SPICE measurements show up to $20 \%$ improvement in the delay time and up to $50 \%$ saving in the total power dissipation comparing with the conventional CMOS bus architecture.
\end{abstract}

\section{INTRODUCTION}

Several low power design techniques have been proposed in order to decrease the total power dissipation of the circuit designs [1-7]. In [3], high 
area is occupied using resistor strings and buffers. In [4], careful design and reference voltage generators are required while in [5], the receiver dissipates static power, and double bus lines are used. Finally, in [6], a method based on diodes without significant decrease in power dissipation is presented. A more efficient technique with high reduction in power dissipation is used in [7] but it leads to a very complex design.

In order to achieve high performance and clock frequency, with an operating power of a few watts, the power dissipated by current designs must be reduced by $1 / 10$. There are two main ways to meet this requirement: 1) By reducing the operating voltage below $1 \mathrm{~V}, 2$ ) By introducing a new circuit design methodology that reduces power dissipation, while keeping a high supply voltage (higher than $2 \mathrm{~V}$ ) [3]. The low swing voltage technique is an example of the second approach. Use of this technique in circuits with large fan-out or high load capacitance results in a significant reduction in the total power dissipation.

Multithreshold technology CMOS circuit designs, which have both high and low threshold voltage transistors in a single chip, can be used to deal with the leakage problem in low voltage, low power, and high performance applications [8]. It can be used in modern VLSI applications in order to increase the power savings and decrease the delay time [9].

Bus is an example where high capacitance has to be driven. It is composed from long interconnections with large fan-out and dissipates in many circuits up to $50 \%$ of the total power dissipation [10]. The purpose of this paper is to introduce new bus circuit designs that reduce power dissipation using multithreshold voltage technology while the operating supply voltage is less than $2 \mathrm{~V}$. In this case low area is observed also using this technique, comparing with other low swing voltage techniques [3-7].

\section{CONVENTIONAL BUS ARCHITECTURE}

The conventional CMOS bus architecture is shown in Fig.1. It consists of a driver, a delay element (interconnection line), and a receiver. The driver and the receiver are both conventional CMOS inverters. The input/output voltage level of the driver/receiver ranges between 0 and the power supply voltage $\left(V_{D D}\right)$. 


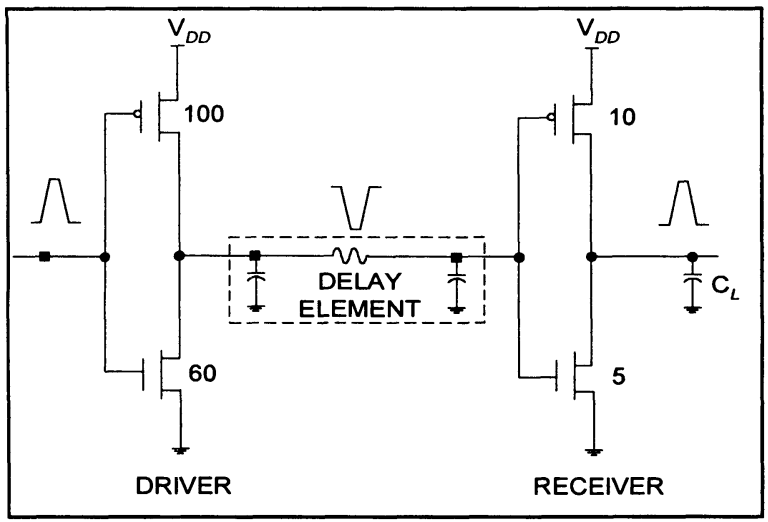

Figure 1. Conventional Bus Architecture

The decrease of the supply voltage is the most efficient way to decrease the power dissipation over the circuits, but on the other hand, it increases the delay time, which makes the new design undesirable in applications where high-speed operation is the main feature. In order to address the problem of high-speed operation in low supply voltage, new devices should be used. Therefore to keep the architecture in high speed-operation and in low supply voltage, the threshold voltage $V_{T}$ of some transistors will certainly be reduced.

\section{NEW BUS DRIVER CIRCUITS}

Several papers have been published using the low-swing voltage technique to decrease the power dissipation over the circuits, but they were introduced the conventional way to decrease the swing voltage by inserting the same threshold voltage of MOSFET transistors. This form of design has the disadvantages of the leakage current, the high used area (inserted MOSFET transistors) and the delay time increase. Not more than $25 \%$ of power delay product reduction can be achieved. Table I shows the number of the added nMOS transistors used in previous designs in order to achieve the low swing voltage in the driver circuit.

Table 1. [Added nMOS Transistors in Previous Low Swing Designs]

Technique

Proposed

[5]
\# of Added Transistor 


\begin{tabular}{cc}
\hline Technique & \# of Added Transistor \\
\hline$[11]$ & 3 \\
{$[12]$} & 3 \\
{$[13]$} & 4 \\
{$[14]$} & 6 \\
{$[15]$} & 15 \\
\hline
\end{tabular}

Fig. 2 shows the proposed Multithreshold Technology CMOS (MTCMOS) driver circuits. The new CMOS inverters are composed by $\mathrm{p} / \mathrm{nMOS}$ transistor with low threshold voltage $\left(\right.$ Low $\left.-\mathrm{V}_{\mathrm{T}}\right)$, as the conventional CMOS driver, and by a new inserted high threshold voltage of $\mathrm{p} / \mathrm{nMOS}$ transistor $\left(\right.$ High- $\left.\mathrm{V}_{\mathrm{T}}\right)$. The inserted transistor almost completely suppresses the leakage current, and decreases the output voltage by its high threshold voltage value.

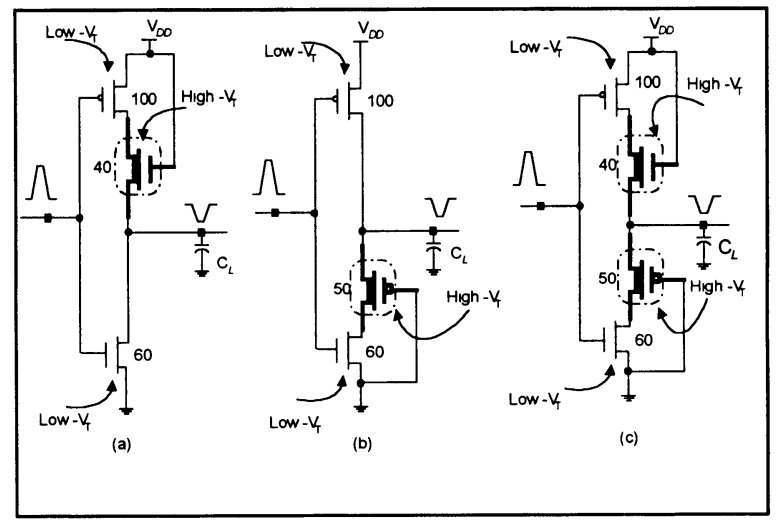

Figure 2. Proposed Multithreshold Technology Low Swing Bus Driver Circuits

According to the direction of the swing voltage reduction in drivers' output, the low swing drivers can be categorized in three different classes. The driver in Fig. 2(a) belongs to the first class called Up Low Swing Voltage Driver (ULD). In this class, the low swing output voltage $\left(\mathrm{V}_{L S}\right)$ ranges between 0 and $\left(V_{D D}-V_{H T N}\right)$ where $\mathrm{V}_{H T N}$ is the threshold voltage of the inserted high threshold nMOS transistor. In order $\mathrm{V}_{L S}$ to drive successfully the succeeding CMOS gate, $V_{D D}-V_{H T N}$ must be always greater than the low threshold of nMOS transistor voltage $V_{L T N}$.

The second class of the low swing voltage drivers called Down Low Swing Voltage Driver (DLD). It is shown in Fig. 2(b). In this class, the low 
swing output voltage $\left(\mathrm{V}_{L S}\right)$ ranges between $V_{H T P}$ and $V_{D D}$, where $\mathrm{V}_{H T P}$ is the threshold voltage of the inserted high threshold pMOS transistor.

The driver in Fig. 2(c) belongs to the third class called Up-Down Low Swing Voltage Driver (UDLD). The output voltage of the driver ranges between $\mathrm{V}_{H T P}$, for $\mathrm{V}_{D D}$ driver input level voltage and $\left(\mathrm{V}_{D D}-\mathrm{V}_{H T N}\right)$, for $O V$ driver input level voltage.

In Table 2, the normalized total power dissipation of the three proposed driver circuits for different supply voltage is compared with the conventional CMOS driver dissipation. The differences of the power dissipation between the three types of the proposed drivers are due to the different threshold voltage values of each high threshold MOSFET transistor. For example, because the threshold voltage value of the inserted high threshold voltage nMOS transistor $(0.55 \mathrm{~V})$ in ULD class is less than the DLD $(|0.65| \mathrm{V})$, it causes the DLD class to dissipate less power than the ULD [16]. The best class is the UDLD because of the lowest output voltage swing, it consists of both high threshold $\mathrm{p} / \mathrm{nMOS}$ transistors.

Table 2. Total Power Dissipation of the Proposed Driver Circuits

\begin{tabular}{ccccc}
\hline Vdd & Conv. & ULD & DLD & UDLD \\
\hline 1 & 1,00 & 0,70 & 0,47 & \\
1,5 & 1,01 & 0,74 & 0,47 & 0,16 \\
1,8 & 1,06 & 0,80 & 0,52 & 0,20 \\
2,1 & 1,23 & 0,93 & 0,63 & 0,24 \\
2,4 & 1,49 & 1,09 & 0,79 & 0,33 \\
2,7 & 1,90 & 1,40 & 1,06 & 0,57 \\
3,0 & 2,50 & 1,93 & 1,50 & 0,95 \\
3,3 & 3,50 & 2,81 & 2,26 & 1,55 \\
\hline
\end{tabular}

When the output voltage swing decreases in the proposed drivers, it causes decreasing in their delay time. Table 3 shows the delay time for the proposed driver circuits compared with the conventional CMOS driver. SPICE measurements are taken with supply voltage $2 \mathrm{~V}$, for the same input slope and load capacitance. The delay time is calculated as the ((rise time + fall time)/2). The transistor sizes (in_m) are shown in Fig. 2.

Table 3. Propagation Delay Time

\begin{tabular}{cc}
\hline Circuit & Propagation Delay Time $(\mathrm{nSec})$ \\
\hline Conventional & 0.173 \\
ULD & 0.170 \\
DLD & 0.163 \\
UDLD & 0.156 \\
\hline
\end{tabular}


Comparisons of the proposed driver circuits with driver circuits proposed in other papers are presented below. The comparison simulation results prove the improvement in power dissipation reduction that achieved by the proposed circuits compared with previous designs.

The driver circuits proposed in [12] and in [20] are compared (Table 4) with the first class (ULD) circuit. All the SPICE measurements are normalized (with the conventional CMOS driver).

Table 4. Normalized Power Dissipation

\begin{tabular}{ccccccc}
\hline Vdd & \multicolumn{3}{c}{ ULD } & \multicolumn{3}{c}{ DLD } \\
\hline & Proposed & {$[12]$} & {$[20]$} & Proposed & Proposed & {$[12]$} \\
\hline 1 & 0,550 & & & 0,435 & & \\
1,5 & 0,552 & 0,600 & 0,660 & 0,438 & 0,116 & 0,170 \\
1,8 & 0,560 & 0,600 & 0,660 & 0,446 & 0,120 & 0,176 \\
2,1 & 0,563 & 0,611 & 0,670 & 0,460 & 0,124 & 0,185 \\
2,4 & 0,578 & 0,622 & 0,689 & 0,487 & 0,137 & 0,204 \\
2,7 & 0,604 & 0,652 & 0,720 & 0,514 & 0,160 & 0,231 \\
3,0 & 0,644 & 0,693 & 0,752 & 0,553 & 0,196 & 0,273 \\
3,3 & 0,693 & 0,737 & 0,807 & 0,602 & 0,254 & 0,320 \\
\hline
\end{tabular}

For the second class (DLD), according to our knowledge no other driver's circuits are proposed in this class. The third class of the low swing voltage drivers UDLD is compared with the driver proposed in [12]. These results proved that the proposed technique using multithreshold voltage technology could operate in low supply voltage (1V) (except UDLD) while the conventional techniques operate in higher supply voltage.

\section{NEW BUS RECEIVER CIRCUITS}

In order to convert the driver low swing output to the full swing level, special receiver designs are required to pull-up the output low swing voltage. When a conventional CMOS inverter is used to convert the low-swing signal to a full-swing signal, the standby power could be important [3].

The proposed receivers are shown in Fig. 3. They are based on the Voltage Sense Transistor Circuit proposed in [11]. They can be implemented with multithreshold technology as the driver designs. For each category of the described drivers, an appropriate corresponding receiver is proposed. 


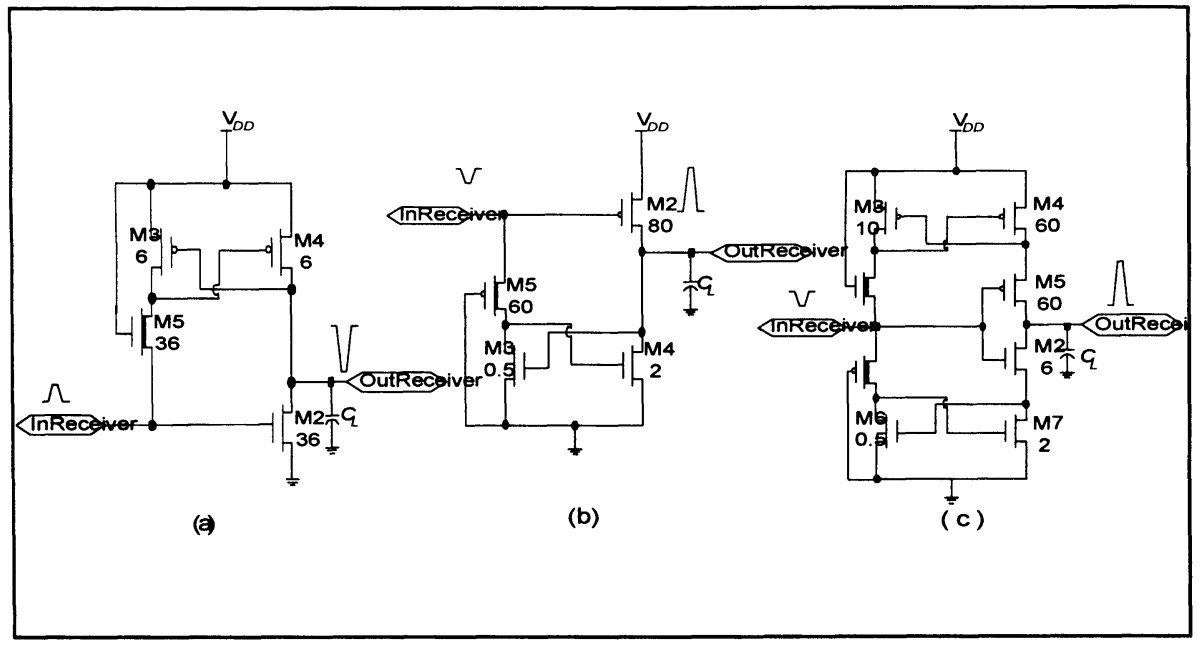

Figure 3. Proposed Receiver Circuits (a) UFR (b) DFR and (c) UDFR

For the first class of drivers, we proposed the Up Full Swing Voltage Receiver (UFR) as shown in Fig. 3(a). The receiver input (InReceiver) is connected to the driver output $\left(V_{L S}\right)$, so the receiver input voltage swings between the values $0 V$ and $\left(V_{D D}-V_{H T N}\right)$. When $V_{L S}=V_{D D}-V_{H T N}$, the transistor M2 turns on, discharging the receiver output node to the ground. Thus, M3 turns on, charging to $V_{D D}$ the gate node of M4, which turns off. The high threshold voltage transistor M5 turns off. When the receiver input is 0 , the transistor M2 turns off while the transistor M5 turns on, discharging the gate of transistor M4 to $0 \mathrm{~V}$. Thus, M4 turns on charging the output load to $V_{D D}$ and ensuring a full swing operation.

The same logic of the previous receiver could be implemented in order to cooperate with the Down Low Swing Voltage Driver. A Down Full Swing Voltage Receiver (DFR) is shown in Fig. 3(b). The logic operation of this receiver is exactly inverse of the UFR. As the receiver input (InReceiver) is $V_{H T P}$, the transistor M2 turns on, charging the receiver output node to the $V_{D D}$. Thus M3 turns on, discharging to GND the gate node of M4, which turns off. The high threshold voltage M5 transistor turns off. When the receiver input is $V_{D D}$, the transistor M2 turns off while the transistor M5 turns on, charging the gate of transistor M4 to $V_{D D}$. Thus, M3 turns off discharging the output load to 0 and ensuring a full swing operation.

The combination of the above proposed receivers results in the Up-Down Full Swing Voltage Receiver (UDFR) as illustrated in Fig.3(c). It is a combination of both previous receivers (UFR, DFR) and a CMOS inverter. When the low swing is from up, the up half receiver converts the low swing to $V_{D D}$, while when the low swing is from down, the down half swing receiver converts the low swing to $O V$. 
Other receiver circuits are proposed in [12], [15] and [17]. They have the common target to pull-up the low swing to full swing voltage. The normalized delay time ((rise + fall)/2) for the different classes are shown in Table 5 . The measurements have been taken by using the same load capacitance and input voltage ramp.

Table 5. Normalized Propagation Delay Time

\begin{tabular}{cccccccccc}
\hline Vdd & \multicolumn{4}{c}{ UFR } & \multicolumn{3}{c}{ DFR } & \multicolumn{3}{c}{ UDFR } \\
\hline & Prop. & {$[12]$} & {$[15]$} & {$[17]$} & Prop. & {$[17]$} & Prop. & {$[12]$} & {$[17]$} \\
\hline 1 & 1,48 & & & & 1,20 & & & & \\
1,5 & 1,42 & & & & 1,21 & 1,34 & 1,69 & & \\
1,8 & 1,36 & 3,10 & 2,38 & 2,80 & 1,23 & 1,36 & 1,67 & 3,50 & 2,84 \\
2,1 & 1,32 & 2,80 & 2,16 & 2,55 & 1,24 & 1,39 & 1,65 & 3,40 & 2,65 \\
2,4 & 1,31 & 2,60 & 2,03 & 2,35 & 1,25 & 1,40 & 1,64 & 3,36 & 2,43 \\
2,7 & 1,30 & 2,50 & 1,94 & 2,22 & 1,29 & 1,45 & 1,61 & 3,31 & 2,32 \\
3,0 & 1,29 & 2,40 & 1,87 & 2,13 & 1,33 & 1,52 & 1,60 & 3,28 & 2,28 \\
3,3 & 1,27 & 2,30 & 1,83 & 2,10 & 1,39 & 1,61 & 1,61 & 3,26 & 2,26 \\
\hline
\end{tabular}

In table 6, the total power dissipation of each proposed receiver comparing with other receivers proposed in previous papers are shown. For the power dissipation measurements, the power meter circuit proposed in [18] has been used.

Table 6. Normalized Power Dissipation

\begin{tabular}{cccccccccc}
\hline Vdd & \multicolumn{4}{c}{ UFR } & \multicolumn{3}{c}{ DFR } & \multicolumn{3}{c}{ UDFR } \\
\hline & Prop. & {$[12]$} & {$[15]$} & {$[17]$} & Prop & {$[17]$} & Prop. & {$[12]$} & {$[17]$} \\
1 & 1,23 & 1,96 & 2,43 & 2,36 & 1,25 & & & 3,5 & 2,95 \\
1,5 & 1,21 & 1,94 & 2,41 & 2,34 & 1,24 & 1,45 & 1,68 & 3,4 & 2,92 \\
1,8 & 1,18 & 1,86 & 2,38 & 2,31 & 1,22 & 1,42 & 1,66 & 3,33 & 2,89 \\
2,1 & 1,16 & 1,83 & 2,34 & 2,29 & 1,21 & 1,40 & 1,64 & 3,25 & 2,87 \\
2,4 & 1,13 & 1,81 & 2,31 & 2,26 & 1,20 & 1,36 & 1,63 & 3,21 & 2,84 \\
2,7 & 1,1 & 1,78 & 2,26 & 2,22 & 1,18 & 1,32 & 1,61 & 3,18 & 2,81 \\
3,0 & 0,98 & 1,76 & 2,25 & 2,18 & 1,160 & 1,293 & 1,59 & 3,12 & 2,76 \\
3,3 & 0,95 & 1,72 & 2,22 & 2,16 & 1,14 & 1,28 & 1,57 & 3,00 & 2,74 \\
\hline
\end{tabular}

The data of the above tables show that using the proposed receivers could pull up the low swing to full swing in low supply voltage (1Volt) (except third class). This advantage is important for future VLSI designs where low supply voltage is required. This advantage could not be achieved using the conventional CMOS technology. We should mention also that the receiver in Fig. 3(c) reduces less power and operates in higher speed than the other receivers mentioned in [12][17], because the output in this class, is the output of the CMOS inverter. This class, the output has sharp edges ranges between 0 and $\mathrm{V}_{D D}$, so less dynamic current dissipation occurred. 
Simulation results of the three proposed bus technique classes (driver/receiver circuits) are shown in Fig. 4. The power delay product of each bus design is normalized with the one of the conventional bus. In all the measurements the same input ramps and load capacitance are used. The results prove, for $1 \mathrm{~V}$ supply voltage, up to $50 \%$ in power delay product reduction could be achieved.

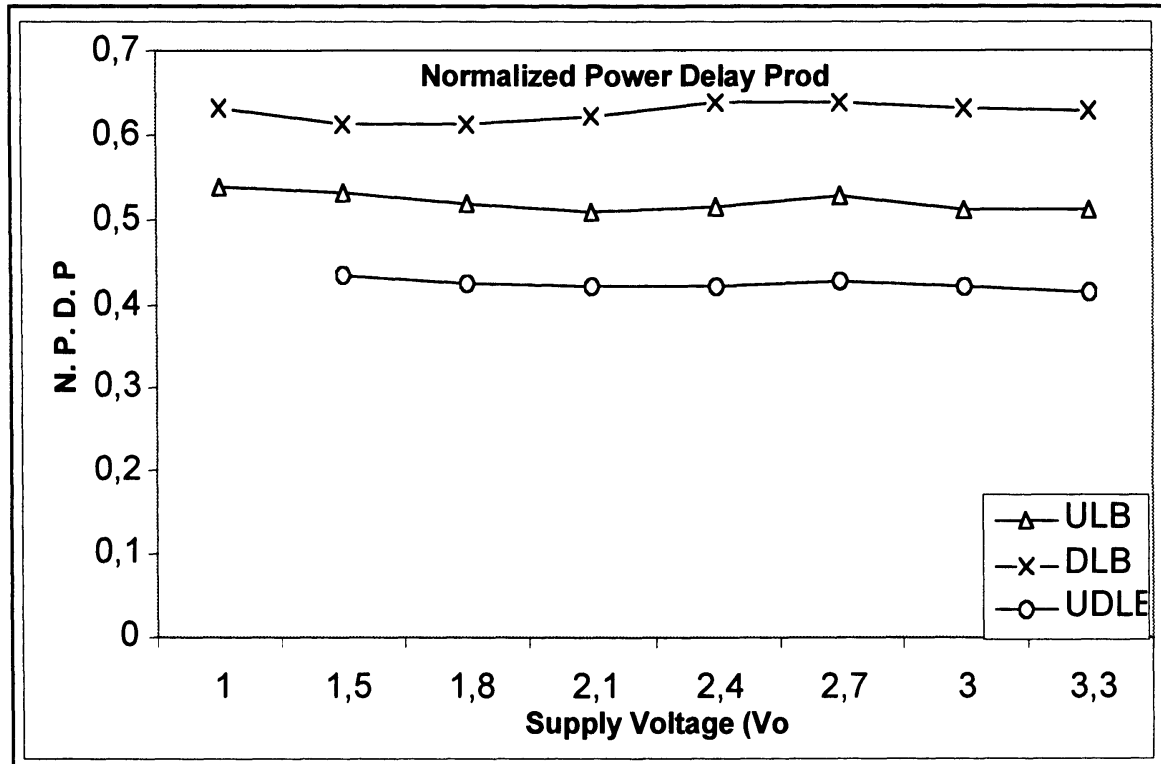

Figure 4. Normalized Power Delay Product for the Three Bus Architecture Classes

\section{NEW BUS REPEATER CIRCUITS}

The delay of a long line with distributed resistive and capacitive components grows as the square of its length. This results in a propagation speed that decreases with line length. To avoid this dependence, a common solution is to separate regularly the interconnection line in segments with the same length, which are driven by repeaters [4].

The line segments could be modelled in different ways. A good approximation of the delay line is obtained with the _ model, achieving accuracy better than $3 \%$ in the delay calculation [4]. Several configurations [19] have been proposed for this architecture. However, these configurations 
operate well in terms of area and delay, but they do not take into account the power dissipation. In order to achieve lower power dissipation on the interconnection lines, low voltage swing repeater designs are proposed. The drivers and receivers, introduced in the previous sections, are used.

Three types of repeaters are presented, corresponding to the driver's classes. For ULD, the Up Low Swing Repeater (ULR) and for DLD, the Down Low Swing Repeater (DLR) are shown in Fig. 5(a) and Fig. 5(b). Merging these two designs a new Up-Down Low Swing Repeater (UDLR) is derived (Fig. 5(c)).

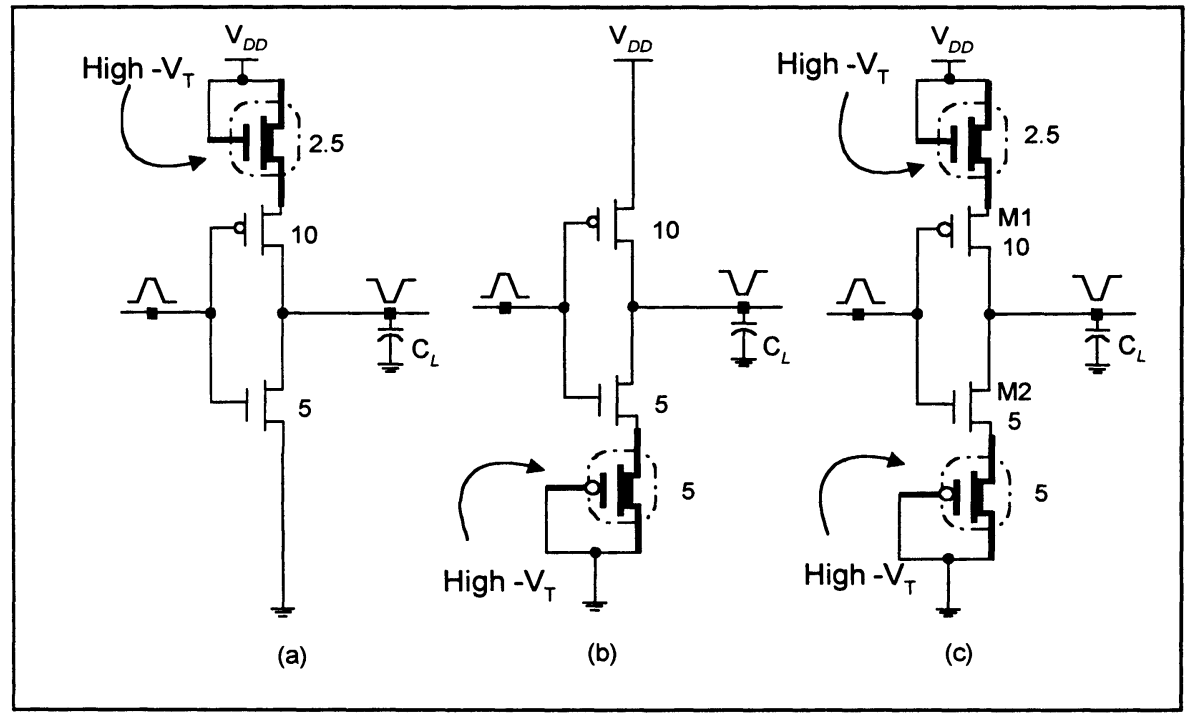

Figure 5. Proposed Repeater Circuits (a) ULR (b) DLR and (c) UDLR

The input of the UDLR (output of the driver) ranges between values $\mathrm{V}_{H T P}$ and $\left(\mathrm{V}_{D D}-\mathrm{V}_{H T N}\right)$. When the input value is $\mathrm{V}_{H T P}$, the pMOS transistor (M1) turns off and the nMOS transistor (M2) turns on (its source voltage value is $\mathrm{V}_{H T P}$ ). The voltage value in the source of transistor $\mathrm{M} 1$ could not exceed the value of $\left(\mathrm{V}_{D D}-\mathrm{V}_{H T N}\right)$, which is the output voltage of the driver. When the input voltage is $\left(\mathrm{V}_{D D}-\mathrm{V}_{H T N}\right)$, the transistor M2 turns off and the transistor M1 turns on (its source voltage value is $\mathrm{V}_{H T N}$ ). The output of the repeater in this case could not exceed the voltage value $\mathrm{V}_{H T P}$.

In order to show the improvement of the different types of the repeater circuits in the delay time as in the power-delay product, we compared each proposed bus type using different number of repeaters and the conventional bus architecture. In the case of conventional bus, it is used conventional driver, $2 p \mathrm{~F}$ capacitor, 0.5 _ resistance, $2 p \mathrm{~F}$ capacitor, conventional receiver, and $2 p \mathrm{~F}$ load capacitance. In the proposed bus, we distributed these values 
according to the number of the repeaters. Table 7 shows the decrement in the normalized delay time, and the decrement in the power delay product for each proposed bus (driver-repeaters-receiver) comparing with the conventional bus (driver-receiver). From Table 7, it is clear that an increase in the number of repeaters causes a decrease in the normalized delay time (as it is expected).

Table 7. Measurments for Different Number of Repeater Circuits

\begin{tabular}{|c|c|c|c|c|}
\hline $\begin{array}{l}\text { BUS } \\
\text { TYPE }\end{array}$ & \# REPEATERS & $\begin{array}{l}\text { NORM. DELAY } \\
\text { TIME }\end{array}$ & $\begin{array}{l}\text { NORM. POWER } \\
\text { DISSIPATION }\end{array}$ & $\begin{array}{c}\text { NORM. POWER } \\
\text { DELAY PRODUCT }\end{array}$ \\
\hline \multirow{4}{*}{ 今 } & 5 & 0.94 & 1.034 & 0.985 \\
\hline & 10 & 0.84 & 1.056 & 0.945 \\
\hline & 15 & 0.63 & 1.083 & 0.855 \\
\hline & 20 & 0.54 & 1.118 & 0.825 \\
\hline \multirow{4}{*}{ 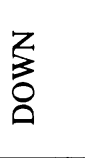 } & 5 & 0.95 & 1.016 & 0.980 \\
\hline & 10 & 0.84 & 1.042 & 0.940 \\
\hline & 15 & 0.62 & 1.089 & 0.850 \\
\hline & 20 & 0.53 & 1.111 & 0.820 \\
\hline \multirow{4}{*}{$\begin{array}{l}Z \\
3 \\
0 \\
0 \\
0 \\
0 \\
0\end{array}$} & 5 & 0.94 & 1.022 & 0.983 \\
\hline & 10 & 0.83 & 1.056 & 0.942 \\
\hline & 15 & 0.64 & 1.073 & 0.854 \\
\hline & 20 & 0.53 & 1.118 & 0.825 \\
\hline
\end{tabular}

The simulation results have been derived by using $0.5-\mathrm{m}$ double metal MTCMOS process. The device parameters are summarized in Table 8.

Table 8. SPICE Parameters multithreshold technology

\begin{tabular}{ccc}
\hline Parameter & High Threshold Voltage & Low Threshold Voltage \\
& Transistor & Transistor \\
\hline Gate Lengh & $0.55 \_\mathrm{m}$ & $0.65 \mathrm{~m}$ \\
Gate Oxide Thickness & $110 \AA$ & $110 \AA$ \\
N-Channel: Vth & $0.55 \mathrm{~V}$ & 0.25 \\
P-Channel: Vth & $-0.65 \mathrm{~V}$ & $-0.35 \mathrm{~V}$ \\
\hline
\end{tabular}

\section{NOISE MARGIN AND MILLER EFFECT}

Noise margin is an important parameter in VLSI design, especially in low swing voltage applications. It appears as problem in long line interconnections and bus designs. The acceptable case of noise margin in either low noise margin or high noise margin is greater than $0.1 \mathrm{~V}_{D D}[10]$. In this paper we refrain from the noise margin problems by using low swing voltage to be $0.35 \mathrm{~V}_{D D}$ and separate the bus by number of repeaters.

The gate-to-drain capacitance effect is called Miller effect, a phenomenon by which a feedback path between the input and the output of 
an electronic device is provided by the interelectrode capacitance. At high frequencies where the gate-to-drain capacitance is not negligible, the circuit is not open but involves a capacitance that is a function of the voltage gain. In the proposed design, for different values of supply voltages in different values of speed operation, Miller effect did not appeared as obstacle in the correct operation of the circuits.

\section{CONCLUSIONS}

Three classes of drivers/receivers for low power design applications are proposed in this paper. They are based on low swing technique using multithreshold voltage technology. In the new driver designs the usage of high threshold voltage transistors causes a significant decrease in the their output swing level. For each proposed driver design, a corresponding receiver circuit is introduced in order to recover the low swing levels. Simulation results show that, by using the proposed architecture, up to $50 \%$ power dissipation savings and $40 \%$ of power delay product reduction could be achieved also. Noise margin and Miller phenomenon have not any effect using the proposed technique.

\section{REFERENCES}

[1] J. M. Rabaey, M. Pedram, "Low Power Design Methodologies," Kluwer Academic Publishers, 1996.

[2] A. P. Chandrakasan, R. W. Brodersen, "Low Power Digital CMOS Design," Kluwer Academic Publishers, 1995.

[3] Y. Nakagome, K. Itoh, M. Isoda, K. Takeuchi, M. Aoki, "Sub-1-V Swing Internal Bus Architecture for Future Low-Power ULSI's," IEEE Journal of Solid-State Circuits, vol. 28, no. 4, pp. 414-419, April 1993.

[4] M. Nekili and Y. Savaria, "Optimal Methods for Driving Long Interconnections in VLSI Circuits," in Proc. IEEE International. Symposium on Circuits and Systems, vol. 4, pp. 343-346, May 1994.

[5] G. Carlo Cardarilli, M. Salmeri, A. Salsano, O. Simonelli, "Bus Architecture for lowpower VLSI digital circuits," in Proc. IEEE International. Symposium on Circuits and Systems, vol. 4, pp. 21-24, May 1996.

[6] Z. Zhu, B. S. Carlson, "Dynamic circuits for CMOS BICMOS low power VLSI design," in Proc. IEEE International. Symposium on Circuits and Systems, vol.??? pp.197200,ISCAS 1996. 
[7] H. Yamauchi, H. Akamatsu, T. Fujita, "An Asymptotically zero power chargerecycling bus architecture for battery-operated Ultrahigh Data Rate ULSI's", IEEE journal of solid-state circuits, vol. 30,pp. 423-431,April 1995.

[8] S. Mutoh, S. Shigematsu, Y. Matsuya, H. Fukuda, T. Kaneko, J. Yamada, "A 1-V Multithreshold-Voltage CMOS Digital Signal Processor for Mobile Phone Application," IEEE Journal of Solid-State Circuits, vol. 31, no. 11, pp. 1795-1801, November 1996.

[9] S. Shigematsu, S. Mutoh, Y. Matsuya, Y. Tanabe, J. Yamada, "A 1-V High-Speed MTCMOS Circuit Scheme for Power-Down Application Circuits, "IEEE Journal of Solid-State Circuits, vol. 32, no. 6, pp. 861-869, June 1997.

[10] N. H. E. West, K. Eshraghian, "Principles of CMOS VLSI Design: A Systems Perspective," Addison-Wesley, 2nd Edition, Reading, 1993.

[11] Rjoub, S. Nikolaidis, O. Koufopavlou, T. Stouraitis, "A New Efficient Low Power Bus Architecture," in Proc. IEEE International. Symposium on Circuits and Systems, vol. 3, pp. 1864-1867, June 1997.

[12] H. Zhang, J. Rabaey, "Low-Swing Interconnect Interface Circuits," in the Proc. IEEE International Symposium on Low Power Electronics and Design, pp. 161-166, June 1998.

[13] M. Hiraki, H. Kojima, H. Misawa, T. Akazawa, Y. Hatano," Data-dependent logic swing internal bus architecture for ultralow-power LSI's," IEEE Journal of Solid-State Circuits, vol. 30, no. 4, pp. 397-402, April 1995.

[14] H. Kojima, S. Tanaka, K. Sasaki, "Half-Swing Clocking Scheme for 75\% Power Saving in Clocking Circuitry," IEEE Journal of Solid-State Circuits, vol. 30, no. 4, pp. 432435, April 1995.

[15] R. Golshan, B. Haroun, " A Novel Reduced Swing CMOS BUS Interface Circuit for High Speed Low Power VLSI Systems," in Proc. IEEE International Symposium on Circuits and Systems, vol. 4, pp. 351-354, May 1994.

[16] A. Rjoub, L. Bisdounis, O. Koufopavlou, "Influence of the nMOS and pMOS Transistor Threshold Voltage on CMOS Circuits Power Dissipation," in proc. IEEE International Conference on Electronics Circuits and Systems, vol. 2, pp. 545-549, 1997.

[17] A. Bellaouar, I. S. Abu-Khader, and M. I. Elmasry, " Low-Power CMOS/BiCMOS Drivers and Receivers for on-Chip Interconnects, "IEEE Journal of Solid-State Circuits, vol. 30, no. 6, pp. 696-700, June 1995.

[18] S. M. Kang, "Accurate Simulation of Power Dissipation in VLSI Circuits," IEEE Journal Solid State Circuits, vol. 21, no. 5, pp.889-891, October 1986.

[19] Sakurai, "Approximation of Wiring Delay in MOSFET LSI," IEEE Journal of SolidState Cicuits, vol. SC-18, pp. 418-426, August 1983. 
[20] H. Kawaguchi, T. Sakurai, “ A Reduced Clock-Swing Flip-Flop (RCSFF) for 63\% Power Reduction," IEEE Journal of Solid-State Circuits, vol. 33, no. 5, pp. 807811,May1998. 